\title{
Genetic linkage map for Amylostereum areolatum reveals an association between vegetative growth and sexual and self-recognition
}

\author{
M.A. van der Nest ${ }^{\mathrm{a}, *}$, B. Slippers ${ }^{\text {a }}$, E.T. Steenkamp ${ }^{\text {b }}$, L. De Vos ${ }^{\text {a }}$, K. Van Zyl ${ }^{\text {a }}$, J. Stenlid ${ }^{\text {, }}$, \\ M.J. Wingfield ${ }^{a}$, B.D. Wingfield ${ }^{a}$ \\ a Department of Genetics, Forestry and Agricultural Biotechnology Institute (FABI), University of Pretoria, Pretoria 0002, South Africa \\ ${ }^{\mathrm{b}}$ Department of Microbiology and Plant Pathology, Forestry and Agricultural Biotechnology Institute (FABI), University of Pretoria, Pretoria, 0002, South Africa \\ ${ }^{\mathrm{c}}$ Department of Forest Mycology and Pathology, Swedish University of Agricultural Sciences, Uppsala, Sweden
}

\section{A R T I C L E I N F O}

Article history:

Received 21 November 2008

Accepted 2 June 2009

Available online $\mathrm{xxxx}$

\section{Keywords:}

Genetic linkage map

mat loci

het loci

Fitness

Balancing selection

\begin{abstract}
A B S T R A C T
Amylostereum areolatum is a filamentous fungus that grows through tip extension, branching and hyphal fusion. In the homokaryotic phase, the hyphae of different individuals are capable of fusing followed by heterokaryon formation, only if they have dissimilar allelic specificities at their mating-type (mat) loci. In turn, hyphal fusion between heterokaryons persists only when they share the same alleles at all of their heterokaryon incompatibility (het) loci. In this study we present the first genetic linkage map for A. areolatum, onto which the mat and het loci, as well as quantitative trait loci (QTLs) for mycelial growth rate are mapped. The recognition loci (mat-A and het- $A$ ) are positioned near QTLs associated with mycelial growth, suggesting that the genetic determinants influencing recognition and growth rate in $A$. areolatum are closely associated. This was confirmed when isolates associated with specific mat and het loci displayed significantly different mycelial growth rates. Although the link between growth and sexual recognition has previously been observed in other fungi, this is the first time that an association between growth and self-recognition has been shown.
\end{abstract}

(c) 2009 Elsevier Inc. All rights reserved.

\section{Introduction}

The sexual and self-recognition systems of fungi and other eukaryotes such as animals and plants have been extensively studied (Nauta and Hoekstra, 1994). In filamentous fungi, sexual recognition is controlled by the genes encoded at the mating-type (mat) loci (e.g., Casselton, 2002), while self-recognition is controlled by the genes encoded at the heterokaryon incompatibility (het) loci (e.g., Worral, 1997; Glass et al., 2000). Although the recognition systems in plants and animals are well-characterized at the genomic and functional levels (Awadalla and Charlesworth, 1999; Meyer and Thomsen, 2001), the recognition systems in fungi have received less attention. Only a few het genes have been characterized at the DNA level and these have been limited to the model ascomycetes Neurospora crassa and Podospora anserina (e.g., Glass et al., 2000; Saupe, 2000). To date, no basidiomycete het genes have been identified. With respect to sexual recognition, the genomic and functional aspects of ascomycete mat loci are relatively well understood, while studies of the mat loci of basidiomycetes have

\footnotetext{
* Corresponding author. Present address: Department of Genetics, Forestry and Agricultural Biotechnology Institute (FABI), 74 Lunnon Road, Hillcrest, Pretoria 0002, South Africa. Fax: +27 124203960.

E-mail address: magriet.vandernest@fabi.up.ac.za (M.A. van der Nest).
}

been limited to the models Schizophyllum commune, Coprinopsis cinerea and Ustilago maydis (e.g., Casselton, 2002).

In this study we considered the sexual and self-recognition systems of the white rot fungus Amylostereum areolatum (Agaricomycetes and Basidiomycotina). A. areolatum lives in a mutualistic association with various woodwasp species (e.g., Sirex noctilio) (Slippers et al., 2003). This partnership is thought to influence the reproductive mode of the fungus, because fungal asexual spores are effectively spread by the woodwasp (Slippers et al., 2003; van der Nest et al., 2008). This asexual mode of reproduction, therefore, serves to explain the overall low genetic heterogeneity of the fungus, as well as the fact that fruiting bodies for $A$. areolatum are rarely found in nature (van der Nest et al., 2008). A. areolatum thus represents a unique model for studying recognition systems as it has an interesting life-cycle that involves a unique interplay between sexual and asexual reproduction.

Sexual recognition in $A$. areolatum is controlled by a tetrapolar mating system where two unlinked mat (mat- $A$ and mat-B) loci determine the outcome of sexual interactions (van der Nest et al., 2008). Homokaryons that are genetically distinct with different allelic specificities at both of their mat loci are sexually compatible and will allow reciprocal nuclear migration after cell fusion to form a heterokaryon (Casselton, 2002). In the agaricomycete model fungi $C$. cinerea and $S$. commune, the mat- $A$ locus encodes homeodomain transcriptional factors, while the mat- $B$ locus 
encodes pheromones and pheromone receptors that control heterokaryon formation (Casselton, 2002). Despite these similarities, the mat loci of $C$. cinerea and $S$. commune differ, especially in terms of the number of sub-loci and the amount of recombination between sub-loci (James et al., 2004). However, little is known for the mat loci of non-model agaricomycetes. This is also true for $A$. areolatum, where knowledge regarding the loci determining sexual recognition is restricted to putative genes that have been identified at the mat- $B$ locus and near the mat- $A$ locus of the fungus (i.e., RAB1 pheromone receptor and MIP mitochondrial intermediate peptidase genes, respectively) (van der Nest et al., 2008).

As is the case in other agaricomycetes, $A$. areolatum has at least two het loci (van der Nest et al., 2008), which is in contrast to the multiplicity of het loci found in ascomycetes. For example, the agaricomycetes Phellinus weiriii, Serpula lacrymans and Heterobasidion annosum have one, two and three/four het loci, respectively (Lind et al., 2007), while N. crassa has at least 11 het loci and P. anserina at least nine het loci (Glass et al., 2000; Saupe, 2000). In general, if two agaricomycete heterokaryons meet in nature, their mycelia will merge, although hyphal anastomosis only persists when the interacting heterokaryons are genetically similar, sharing identical alleles at all of their het loci (Worral, 1997). When the interacting heterokaryons have different alleles at some or all of their het loci, cell death prevents anastomosis and a zone of inhibition forms between the interacting heterokaryons. In general, it is thought that vegetative incompatibility plays a role in preserving genetic identity and preventing the spread of mycoviruses, debilitated organelles and deleterious plasmids (Worral, 1997). However, the precise biological significance of this phenomenon is still largely unclear, and a deeper understanding of how and why vegetative incompatibility evolved, will require comparative studies of the het loci of many diverse fungi.

Our overall goal was to establish a genetic foundation from which to eventually study the structure, organization and evolution of the recognition loci of $A$. areolatum. This entailed construction of a genetic linkage map for $A$. areolatum and positioning of its recognition loci, as have been done for other eukaryotes (e.g., Kubisiak and Milgroom, 2006; Lind et al., 2007; Leppälä et al., 2008). For this purpose we used amplified fragment length polymorphisms (AFLPs) (Vos et al., 1995) for map construction and AFLPbased bulked segregant analysis (BSA) to identify markers closely linked to the recognition loci. We also compared marker distribution and segregation ratio distortions associated with markers linked to the recognition loci, with those associated with the rest of the genome. This was particularly relevant because it has previously been shown that the recognition loci are subject to evolutionary forces that are markedly different from those acting on the rest of the genome (e.g., Meyer and Thomsen, 2001; Takebayashi et al., 2004; Uyenoyama, 2005). A final objective was to use the map to reveal a possible association between the recognition loci and the genes involved in fitness, as has been shown for other agaricomycetes (e.g., Callac et al., 1997; Larraya et al., 2001, 2002; Olson, 2006). This was accomplished by identifying putative quantitative trait loci (QTLs) linked to mycelial growth rate as a measure of fitness (Hill and Otto, 2007) and determining whether or not they are associated with the recognition loci of $A$. areolatum.

\section{Materials and methods}

\subsection{Isolates and growth conditions}

Eighty homokaryotic isolates that were obtained from an $A$. areolatum basidiocarp (van der Nest et al., 2008) collected in Austria were used for segregation analysis. Working cultures of the parent strain (CMW16848) and homokaryons (CMW16848 ${ }_{[1-80]}$ ) were maintained on pine extract medium as previously described (van der Nest et al., 2008).

\subsection{AFLP and BSA analysis}

DNA was isolated from cultures (van der Nest et al., 2008) and stored at $-20^{\circ} \mathrm{C}$. The AFLP procedure described by De Vos et al. (2007) using 20 primer pairs was followed and AFLP fragments were visualized using the 4200 Li-COR ${ }^{\circledR}$ automated DNA sequencer as described by Myburg et al. (2001). To ensure that the AFLP profiles used in this study were reproducible, we repeated the AFLP procedure using DNA isolated from eight individuals. AFLP fragments were scored as present (coded as ' $A$ ') or absent (coded as ' $\mathrm{H}$ ') using QUANTAR Version 1.0 (KeyGene Products B.V., The Netherlands). Each fragment that segregated in the progeny was treated as an independent genetic locus. As previously proposed (Bagley et al., 2001), we ensured that our scoring of the polymorphic bands segregating in the progeny was repeatable, by excluding bands with low intensity, bands with very high and low molecular weight and bands that were inordinately close to each other and thus complicating their accurate scoring. Bands were also rescored manually several times to ensure that they had been scored correctly. The mat and het alleles that segregated in the mapping population were identified in a previous study using hyphal morphology and DNA-based assays (van der Nest et al., 2008). It is possible that additional het loci exist in $A$. areolatum, but we could only identify two het loci segregating in the mapping population. The two mat and the two het loci were each treated as four markers segregating in the progeny.

BSA was used to identify additional AFLP markers that were closely linked to any of the mat or het loci. For this purpose eight AFLP template pools consisting of equal amounts of DNA from 15 different progeny sharing unique mat or het alleles were subjected to AFLP analysis. Templates 1, 2, 3 and 4 contained DNA from progeny sharing the mat-A1, mat-A2, mat-B1 and mat-B2 alleles, respectively. Templates 5, 6, 7 and 8 contained DNA from progeny sharing the het- $A 1$, het-A2, het-B1 and het-B2 alleles, respectively. Thirty one AFLP primer pairs were tested on the eight pools to identify possible markers linked to the loci of interest. Four primer pairs that generated fragments potentially linked to any of the mat or het loci were chosen for analysis in the entire mapping population. These fragments were scored as described above and included during map construction.

\subsection{Framework map construction}

JoinMap $^{\circledR} 3.0$ (Van Ooijen and Voorrips, 2001) was used for map construction and for graphical representation of the linkage groups. This program uses likelihood of odds (LOD) scores of recombination frequencies to assemble pairwise marker associations and then constructs maps of these groupings using a modified squares method, in which the squares of the LOD scores are used as weights (Van Ooijen and Voorrips, 2001). The Kosambi mapping function (Kosambi, 1944) was used to convert recombination fractions into map distances. Since it was not possible to distinguish between the parental genotypes that generated the original basidiocarp or heterokaryon, the data were coded as HAP, i.e., haploid originating from a diploid parent with unknown linkage phase. Initial linkage groups were determined based on a LOD threshold value between 2.0 and 10.0 and a recombination ratio upper threshold of 0.45 in the pairwise comparison. The LOD scores used by JoinMap are based on $\chi^{2}$ tests for independence of segregation and markers with high $\chi^{2}$-contribution to a linkage group were excluded from the analysis as they could have resulted in a poor fit and a significant disturbance of map order across the linkage group (Staelens et al., 2008). All the markers, including those showing segregation distortion $(\alpha=0.05)$, were included and evaluated during linkage map construction. 
The total genome length of the framework map was estimated using the Hulbert estimate, $E(G)=n(n-1) X / K$, where $n$ is the number of loci, $X$ is the maximum map distance between locus pairs above a minimum LOD value for linkage grouping $(Z)$, and $K$ is the number of locus pairs above $Z$ (Hulbert et al., 1988). The linkage threshold of LOD $3.0(Z)$ was used to estimate genome length as the mapping set was separated into different linkage groups at this threshold. For a $Z$-value of 3 , both $X$ and $K$ were obtained from the list of values generated by the 'big Lods' command of the program MAPMAKER Macintosh V2.0 (Lander et al., 1987). Theoretical genome coverage was estimated using the formula, $C=1-e^{-2 d n / L}$, where $C$ is the proportion of the genome within $d \mathrm{cM}$ (centimorgan) of a marker, $L$ is the estimated genome length and $n$ is the number of markers (Remington et al., 1999).

\subsection{Marker distribution and haplotype analysis}

Marker distribution between different linkage groups was evaluated by comparing the actual marker density with what would be expected under a Poisson distribution (Remington et al., 1999). For this purpose, each linkage group had a length $G_{i}=M_{i}+2 s$, where $M_{i}$ is the map distance between terminal markers of the ith linkage group, and $s$ is the average marker spacing calculated by dividing the summed length of all maps by the number of marker intervals. Where the underlying marker density was the same for all linkage groups, the number of markers $\left(m_{i}\right)$ in the $i$ th linkage group would be a sample from a Poisson distribution with parameter, $\lambda_{i}=m G_{i} \mid$ $\Sigma_{i} G_{i}$, where $m$ is the total number of markers. Here, the null hypothesis requires that there is equal marker density in each of the linkage groups (Remington et al., 1999).

The number of crossovers per linkage group per individual was estimated by visualizing the haplotypes of the individuals using the Graphical GenoTyping software (GGT) (Van Berloo, 1999) and counting the number of observed crossovers. Double-crossovers flanking a single marker-locus were not included in the estimate because their occurrence within short intervals could suggest genotyping errors or they could have resulted from gene conversion during local DNA repair (Shibata, 2001). The distribution of crossovers in the 10 major linkage groups were examined by performing a two-tailed test in Statistica Version 7.1 (www.statsoft.com) to determine whether the distribution of crossovers in the linkage groups follow a Poisson distribution.

\subsection{Mycelial growth estimation and QTL identification}

The mycelial growth rate of the 80 homokaryotic $A$. areolatum isolates used in this study was determined by taking a plug of mycelium from the edge of an actively growing culture on potato dextrose agar (Biolab, Johannesburg, South Africa) and placing these at the centres of $90 \mathrm{~mm}$ Petri dishes. After 7 days of incubation at $25^{\circ} \mathrm{C}$ in the dark, the radial extension of each colony was measured along two perpendicular axes. Average colony diameter, standard deviation and analysis of variance (ANOVA) were determined using Statistica. Three repetitions per progeny were made with the mean mycelial growth based on six measurements being used for QTL analysis. Individual observed broad-sense heritability $\left(H^{2}\right)$ which serves as an indication of the extent to which mycelial growth of individuals is linked to a genotype, were determined using the equation $H^{2}=\sigma_{\mathrm{G}}^{2} / \sigma_{\mathrm{p}}^{2}$, where $\sigma_{\mathrm{G}}^{2}=$ genotypic variance and $\sigma_{\mathrm{p}}^{2}=$ phenotypic variance (Falconer, 1989).

Map Manager QTX version b20 (Manly et al., 2001) was utilized to identify markers significantly associated with mycelial growth rate at $25^{\circ} \mathrm{C}$. Markers with segregation distortion were included in all of the analyses, using the Map Manager QTX "allow for segregation distortion" function. Significance levels for highly significant, significant and suggestive QTLs were determined with 1000 permutations using $1 \mathrm{cM}$ steps. Simple interval mapping (SIM) was used to determine the likelihood for the presence of a segregating QTL for every $1 \mathrm{cM}$ of the $A$. areolatum linkage map, while composite interval mapping (CIM) was used to establish the effect of QTL combinations. Likelihood ratio statistic (LRS) values were converted into LOD values by a direct transformation using the equation LRS $=4.6 \times$ LOD (Liu, 1998). Primary QTLs were identified when SIM and CIM values coincided and exceeded the permuted test statistic thresholds for SIM, while secondary QTLs were identified when either the SIM or CIM scan exceeded the permuted SIM test statistic threshold (Thomas et al., 1998). Statistica was used to perform $t$-tests to determine if there were significant differences in mycelial growth of homokaryons that have different alleles at the loci associated with QTLs for mycelial growth.

\section{Results}

\subsection{AFLP and BSA analysis}

AFLP analysis was performed on DNA of the A. areolatum parent heterokaryon, as well as the 80 selected homokaryons using 20 different primer combinations (Table 1). Of the 31 AFLP primer combinations evaluated for BSA, only four (Table 1) generated AFLP fragments that were potentially linked to recognition loci. The analysis of these on the entire mapping population revealed five markers that apparently co-segregated with the loci of interest. One of these AFLP markers (at14399) segregated with the het-A locus and one (ac16085) with the het- $B$ locus. One AFLP marker (cc13153) segregated with mat- $A$, while two markers (ac10290 and ac16260) segregated with the mat- $B$ locus. However, none of these markers co-segregated consistently with the various recognition loci in the progeny.

For each of the 24 primer combinations used, an average of 10 polymorphic AFLP bands was identified, with a total of 245

Table 1

The number of monomorphic and polymorphic fragments per primer combination.

\begin{tabular}{llcc}
\hline $\begin{array}{l}\text { EcoRI } \\
\text { primer }^{\text {a }}\end{array}$ & $\begin{array}{l}\text { MseI } \\
\text { primer }^{\text {b }}\end{array}$ & $\begin{array}{l}\text { Monomorphic bands/ } \\
\text { primer }\end{array}$ & $\begin{array}{l}\text { Polymorphic bands/ } \\
\text { primer }\end{array}$ \\
\hline E-AA & M-GC (11) & 9 & 4 \\
E-AA & M-AT (2) & 20 & 11 \\
E-AA & M-AG (3) & 24 & 8 \\
E-AA & M-AC (4) & 19 & 11 \\
E-AA & M-GA (9) & 14 & 11 \\
E-AC & M-AT (2) & 10 & 10 \\
E-AC & M-TT (6) & 10 & 10 \\
E-AT & M-TT (6) & 17 & 13 \\
E-AT & M-GC (11) & 10 & 17 \\
E-CC & M-AT (2) & 18 & 11 \\
E-CC & M-AC (4) & 19 & 11 \\
E-CC & M-TT (6) & 10 & 12 \\
E-CC & M-GC (11) & 9 & 9 \\
E-CT & M-TT (6) & 8 & 14 \\
E-CT & M-GC (11) & 12 & 13 \\
E-TC & M-AA (1) & 16 & 18 \\
E-TC & M-AT (2) & 13 & 14 \\
E-TC & M-AG (3) & 15 & 11 \\
E-TC & M-AC (4) & 17 & 7 \\
E-AT & M-CA (13) & 19 & 10 \\
BSA & & & 3 \\
E-AT & M-CT (14) & 8 & 9 \\
E-CC & M-CA (13) & 8 & 3 \\
E-AC & M- GT(10) & 18 & 13 \\
E-AC & M-CC (16) & 10 & \\
\hline
\end{tabular}

a The EcoRI adapter primer sequence without selective nucleotides was 5-GACTGCGTACCAATTC-3'

b The Msel adapter primer sequence without selective nucleotides was 5-GATGAGTCCTGAGTAA-3'. MseI primer numbers are indicated in parentheses. 
polymorphic bands across all primer combinations (Table 1$)$. The primer pair EcoRI $(+\mathrm{TC})+\mathrm{MseI}(+\mathrm{AA})$ gave the largest number of polymorphic AFLP fragments (18), while the primer pairs EcoRI $(+A T)+\operatorname{MseI}(+C T)$ and $\operatorname{EcoRI}(+A C)+\operatorname{MseI}(+C C)$ gave the smallest number of polymorphic AFLP fragments (3) (Table 1). Identical profiles were generated when the AFLP procedure was repeated on DNA isolated from eight selected progeny, supporting previous views regarding the repeatability of AFLPs (Myburg et al., 2001).

\subsection{Framework map construction}

JoinMap $^{\circledR} 3.0$ generated a framework map consisting of 151 AFLP markers (including those identified using BSA) distributed over 10 major linkage groups with more than seven markers (Fig. 1). Another 49 linked AFLP markers mapped to 15 minor linkage groups containing 2-6 loci. Of the segregating markers, $81.6 \%$ mapped to the 10 major and 15 minor linkage groups, while $18.4 \%$ (45) of the markers did not map to any of the linkage groups. The 10 major linkage groups ranged in size from $164 \mathrm{cM}$ (linkage group LG1) to $65 \mathrm{cM}$ (linkage group LG10). The four recognition loci (mat- $A$ and mat- $B$; het- $A$ and het- $B$ ) mapped on separate linkage groups. As expected, the specific marker(s) identified using BSA were also closely linked to the respective recognition loci. The total observed length of the map, when only including the 10 major linkage groups with 151 AFLP markers and the four recognition loci, was $1046 \mathrm{cM}$ with an average distance between markers of $7.2 \mathrm{cM}$ (Table 2). The largest mapped distance between markers was $26 \mathrm{cM}$ for linkage group LG3. The total observed length of the map including the minor and major linkage groups with 204 markers was $1693 \mathrm{cM}$ with an average distance between markers of $8.3 \mathrm{cM}$.

Genome length (Hulbert et al., 1988) was estimated as $2922.6 \mathrm{cM}$. Based on this estimate, the theoretical genome coverage was calculated where $67.2 \%$ of the markers were $\leqslant 10 \mathrm{cM}$ apart and $89.3 \%$ of the markers were $\leqslant 20 \mathrm{cM}$ apart (Table 2 ). Because the physical genome size of $A$. areolatum is unknown, the genome size (33.7 Mbp) of a related fungus, $H$. annosum (http://genome.jgipsf.org), was used. Accordingly, the physical distance per unit of recombination was estimated as $32.2 \mathrm{kbp} / \mathrm{cM}$ for the major linkage groups and $19.9 \mathrm{kbp} / \mathrm{cM}$ for all of the linkage groups. With the physical distance per genetic distance estimated as $19.9 \mathrm{kbp} / \mathrm{cM}$, marker at14399 would theoretically be positioned $119.4 \mathrm{kbp}$ from locus het- $A$, and marker ac16085 would be positioned $119.4 \mathrm{kbp}$ from locus het- $B$. Markers cc13153 and aa10340 would be positioned $99.5 \mathrm{kbp}$ and $59.7 \mathrm{kbp}$, respectively, from locus mat- $A$, while marker ac10290 would be positioned $119.4 \mathrm{kbp}$ from locus mat- $B$. With the exception of aa10340 all these markers were identified using BSA.

\subsection{Marker distribution and haplotype analysis}

$\chi^{2}$-analysis indicated that $29.2 \%$ of the AFLP markers ( 44 of the 151 markers) that were placed on the map, showed significant segregation distortion $(\alpha=0.05)$ as they did not segregate in a Mendelian fashion (Fig. 1). Despite the fact that segregation distortion can interfere with mapping (Hackett and Broadfoot, 2003), these markers were retained in the map as their removal would have significantly reduced the map coverage.

Markers with segregation distortion mapped evenly to all of the linkage groups of $A$. areolatum, with several of these markers clustering together in the same region of a specific linkage group (Fig. 1). Although the distorted markers mapped to several linkage groups, LG7 had an excess of markers (6 of 44) showing segregation distortion. While the recognition loci were positioned near markers displaying segregation distortion, the alleles present at the mat and het loci did not demonstrate significant $(\alpha=0.05)$ segregation distortion. Furthermore, it was not possible to associate segregation distortion to a specific parent, because the genotypes of the parental strains are not known.

The two-tailed tests showed that there were significant differences (at $\alpha=0.05$ ) in marker density in two of the 10 major linkage groups (Table 3). Linkage group LG1 had a greater number of markers, while linkage group LG7 had fewer markers than expected under a Poisson distribution where the null hypothesis is that the average marker spacing $(7.2 \mathrm{cM})$ is the same for all linkage groups. There were 23 and 15 markers $\left(\lambda_{i}\right)$ expected to be located on linkage groups LG1 and LG7, respectively. However, 35 and eight markers $\left(m_{i}\right)$, respectively, were actually observed on these linkage groups. Linkage group LG1, therefore, has significantly more markers and it also contained a high number of markers displaying segregation distortion.

Haplotype analysis of the individual genotypes using GGT revealed no linkage distortion in any of the 10 major linkage groups identified for $A$. areolatum, with both the inferred parental genomes segregating in a 1:1 ratio. Haplotype analysis of the individual genotypes was also used to estimate the number of crossover events per linkage group per individual. There were, however, no significant differences (at $\alpha=0.05$ ) in the number of crossovers in the 10 major linkage groups, with all of these groups following a distribution as expected under a Poisson model. The average number of crossover events per linkage group per individual was 1.06, with linkage group LG10 displaying the fewest crossovers (0.66) and linkage group LG1 displaying the most crossovers (1.90) (Table 4). The low number of observed crossovers was probably due to our underestimation of linkage group sizes, as this number is dependent on linkage group size and the linkage groups identified in this study probably do not cover full chromosomes.

\subsection{Mycelial growth estimation and QTL identification}

Mycelial growth rate of the A. areolatum homokaryotic progeny and their heterokaryotic parent were determined. The average mycelial growth rate of the mapping population was $58.8 \mathrm{~mm} /$ wk with a standard deviation of $17.3 \mathrm{~mm} /$ wk after incubation at $25^{\circ} \mathrm{C}$. The ANOVA showed that the average mycelial growth rate amongst the homokaryons differed significantly (Table 5). The distribution of mycelial growth rate was continuous at $25^{\circ} \mathrm{C}$, with $25 \%$ of the fastest growing homokaryons having the same mycelial growth rate $(70-80 \mathrm{~mm} / \mathrm{wk})$ as the parent. The frequency distribution of mycelial growth rate of the homokaryons was binomial, with two distinct peaks at growth rate classes $25-30 \mathrm{~mm} / \mathrm{wk}$ and $70-75 \mathrm{~mm} /$ wk (Fig. 2). The broad-sense heritability $\left(H^{2}\right)$ of mycelial growth rate was estimated as 0.98 . Although the individual heritability of mycelial growth rate for the individual homokaryons used in this study was very high, it is similar to the individual heritability of mycelial growth in H. annosum (0.97) (Olson, 2006). Olson (2006) suggested that this high value might be due to low environmental variation in the experiment.

Using SIM, a total of seven putative QTLs associated with mycelial growth in $A$. areolatum were identified and located on linkage groups LG2, LG4 and LG5 (Fig. 1; Table 6). Based on 1000 permutations using $1 \mathrm{cM}$ steps, Map Manager QTX indicated that LOD scores between 2.4 and 4.1 represent significant QTLs, while LOD scores between 1.1 and 2.4 represent suggestive QTLs. Only the putative QTL positioned on LG2 in the same region as the het- $A$ locus (i.e., $0 \mathrm{cM}$ from the het- $A$ locus, explaining $16 \%$ of the phenotypic variance) was significant ( $\mathrm{LOD}>2.4$ ). The other six putative QTLs were suggestive (LOD 1.1-2.4) and were positioned on linkage groups LG2, LG4 and LG5. Using CIM, only six putative QTLs associated with mycelial growth were detected. The second putative QTL on linkage group LG2 was not included because its LOD 
LG1

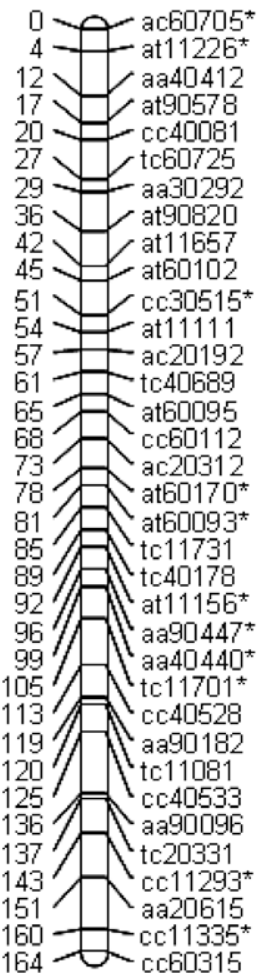

LG 6

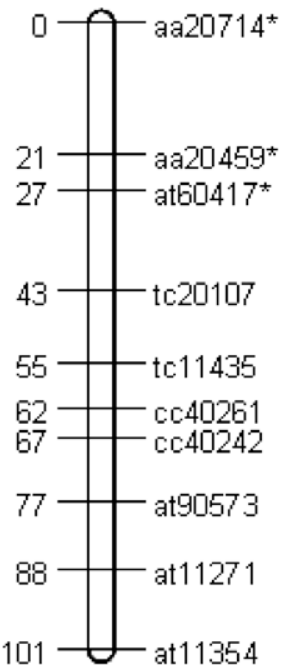

LG 2

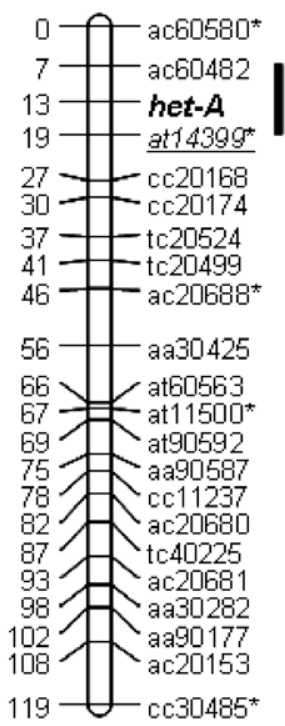

LG 7

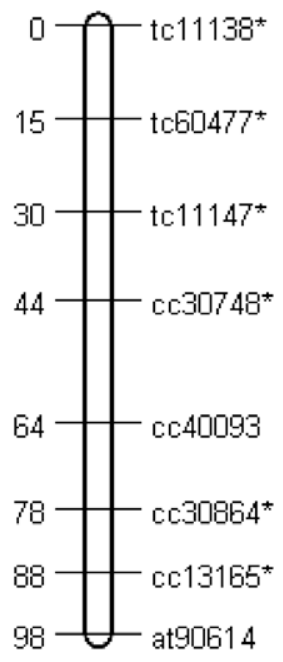

LG 3

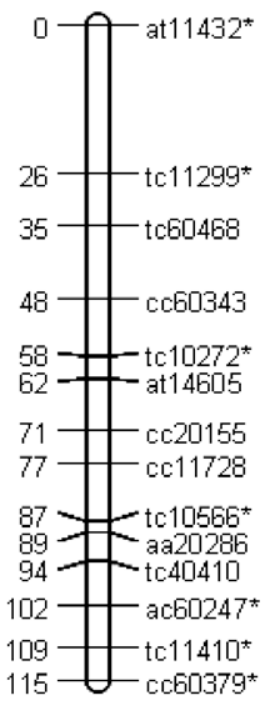

LG 8

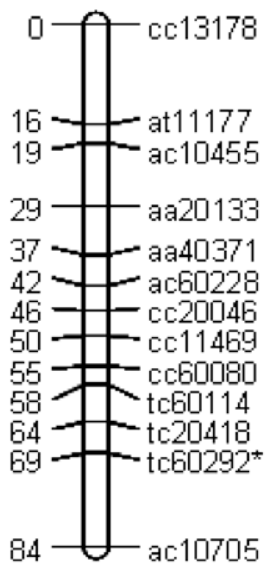

LG 4

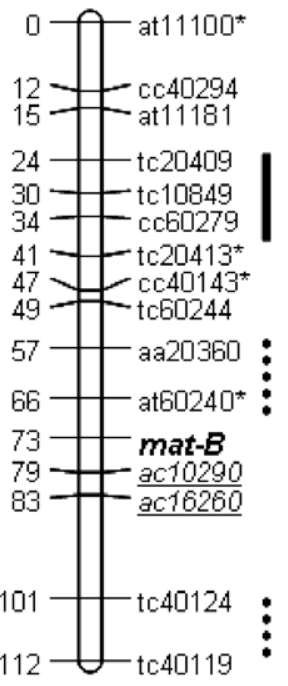

LG 5

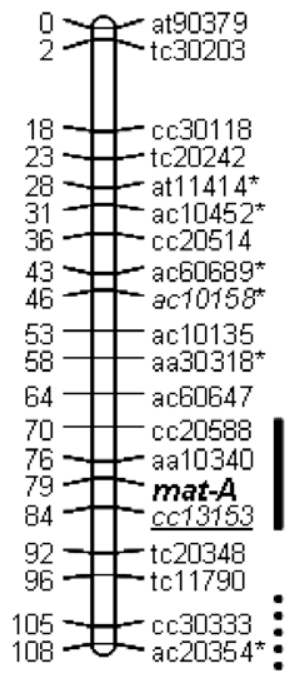

LG 9

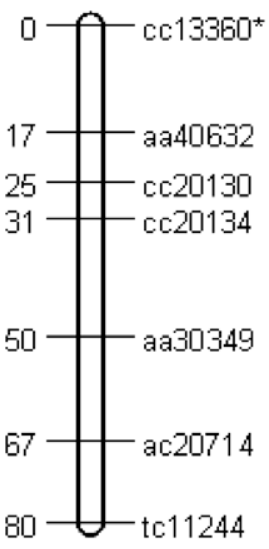

LG 10

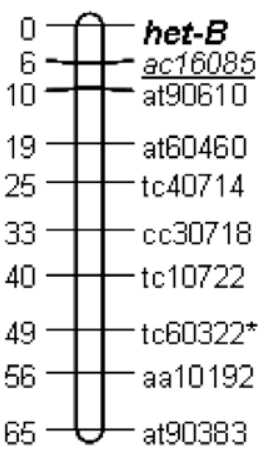

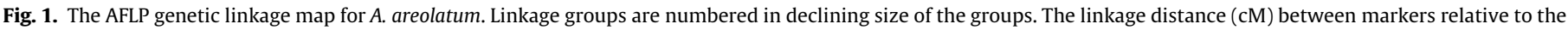

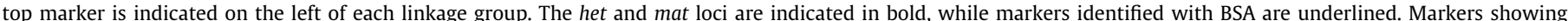

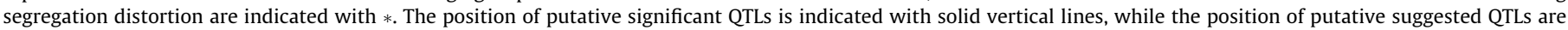

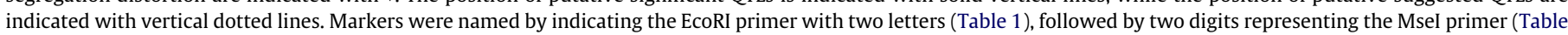
1) and then three digits that represent the size of the fragment in base pairs.

score was below the suggested significance level. However, the LOD values of two of the QTLs increased from suggestive to the significant level using CIM. These are the QTL positioned in the same region as the mat-A locus on linkage group LG5 (i.e., $1 \mathrm{cM}$ from the mat- $A$ locus, explaining $14 \%$ of the phenotypic variance) and the QTL positioned in the same genomic region as the AFLP marker tc10849 (i.e., 3 cM from the tc10849 locus, explaining 11\% of the phenotypic variance). One of the suggestive QTLs was also positioned in the same genomic region as the mat- $B$ locus (i.e., $6 \mathrm{cM}$ from the mat- $B$ locus, explaining $6 \%$ of the phenotypic variance). All of the putative QTLs detected with CIM, explained 76\% of the total mycelial growth variation.

The average growth for homokaryons with different alleles at their het- $A$ and mat- $A$ loci differed significantly $(P<0.05)$ (Table 
Table 2

Summary of map constructed using AFLP markers, mating-type and vegetative compatibility information for a set of Amylostereum areolatum progeny.

\begin{tabular}{ll}
\hline Description & $\begin{array}{l}\text { Amylostereum areolatum } \\
\text { map }\end{array}$ \\
\hline Markers & 200 \\
Number of framework markers selected & 45 \\
Number of unlinked markers & 44 \\
Number of markers showing transmission ratio & \\
$\quad$ distortion & \\
Framework map & 10 \\
Number of major linkage groups & 15 \\
Number of minor linkage groups & 112.9 \\
Average linkage group size $(\mathrm{cM})^{\mathrm{b}}$ & 8.3 \\
Average marker spacing (cM) & 1693 \\
Observed map length $(\mathrm{cM})$ & 19.9 \\
Physical distance per unit of recombination $(\mathrm{kb} / \mathrm{cM})^{\mathrm{d}}$ & \\
Estimate of genome length & 2922.6 \\
Using Hulbert estimate of genome length $(\mathrm{cM})^{\mathrm{e}}$ & \\
Framework map coverage & \\
Map coverage $(c=100 \%)$ at $d=20 \mathrm{cM}(\%)$ & 89.3 \\
Map coverage $(c=100 \%)$ at $d=10 \mathrm{cM}(\%)$ & 67.2
\end{tabular}

${ }^{a}$ Departure of markers from the expected $1: 1$ ratio (at the $5 \%$ level of significance).

b Distance are in centimorgans (cM).

c Calculated by dividing the summed length of all the linkage groups by the number of marker intervals. The number of marker intervals was calculated by the number of linked markers minus the number of linkage groups.

d The genome size of $H$. annosum (33.7 Mb) (http://genome.jgi-psf.org/) was used to calculate the physical distance per unit of recombination.

e The total genome length of the framework map was estimated as described by Hulbert et al. (1988).

$\mathrm{f}$ The theoretical genome coverage was estimated according to Remington et al. (1999).

Table 3

Verification of random marker distribution using Poisson two-tailed P-test. ${ }^{a}$

\begin{tabular}{llllll}
\hline $\begin{array}{l}\text { Linkage } \\
\text { group }\end{array}$ & $\begin{array}{l}\text { Number of } \\
\text { markers }\left(m_{i}\right)\end{array}$ & $\begin{array}{l}\text { Map } \\
\text { length } \\
\left(M_{i}\right)\end{array}$ & $\begin{array}{l}\text { Inferred } \\
\text { map length } \\
\left(G_{i}\right)\end{array}$ & $\begin{array}{l}\text { Expected } \\
\text { number of } \\
\text { markers }\left(\lambda_{i}\right)\end{array}$ & $\begin{array}{l}\text { Poisson } \\
\text { two-tailed } \\
P \text {-value }\end{array}$ \\
\hline LG 1 & 35 & 164 & 178.4 & 23.5 & $0.0059^{*}$ \\
LG 2 & 22 & 119 & 133.4 & 16.4 & 0.0358 \\
LG 3 & 14 & 115 & 129.4 & 16.9 & 0.0814 \\
LG 4 & 16 & 112 & 126.4 & 16.5 & 0.0985 \\
LG 5 & 20 & 108 & 122.4 & 19.9 & 0.0888 \\
LG 6 & 10 & 101 & 115.4 & 15.0 & 0.0486 \\
LG 7 & 8 & 98 & 112.4 & 14.6 & $0.0234^{*}$ \\
LG 8 & 13 & 84 & 98.4 & 12.8 & 0.1098 \\
LG 9 & 7 & 80 & 94.4 & 12.3 & 0.0385 \\
LG 10 & 10 & 65 & 79.4 & 10.3 & 0.1246 \\
\hline
\end{tabular}

a Poisson probability of having as many markers as the observed number of markers, $m_{i}$ in the $i$ th linkage group under the null hypothesis that the average marker spacing $(7.1 \mathrm{cM})$ is the same for all linkage groups.

b In a two-tailed test, a $P$-value of 0.025 corresponds to a significance level of 0.05 . Linkage groups 1 and 7 (indicated with $*$ ) shown significant deviation from the Poisson distribution.

5). Homokaryons with alleles het-A2 and mat-A1 generally grew faster, while homokaryons with alleles het-A1 and mat-A2 grew slower. The mean mycelial growth for homokaryons with different alleles at the tc10849 locus also differed significantly $(P<0.05)$. Homokaryons that share the tc10849-1 allele displayed higher average mycelial growth rate than homokaryons that share the tc10849-2 allele. The average mycelial growth rate for homokaryons with different alleles at their het- $B$ and mat- $B$ loci did not differ significantly $(P>0.05)$.

No epistatic interactions between the QTLs or unlinked markers were detected using the "interactions" function in Map Manager QTX, thereby suggesting that these loci act independently.
Table 4

Distribution of observed crossovers per linkage group.

\begin{tabular}{lllll}
\hline $\begin{array}{l}\text { Linkage } \\
\text { group }\end{array}$ & $\begin{array}{l}\text { Number of } \\
\text { markers }\end{array}$ & $\begin{array}{l}\text { Map } \\
\text { length } \\
\text { (cM) }\end{array}$ & $\begin{array}{l}\text { Average marker } \\
\text { interval }(\mathrm{cM})^{\mathrm{a}}\end{array}$ & $\begin{array}{l}\text { Number of } \\
\text { crossover events }^{\mathrm{b}}\end{array}$ \\
\hline LG 1 & 35 & 164 & 5.0 & 1.90 \\
LG 2 & 22 & 119 & 5.4 & 1.40 \\
LG 3 & 14 & 115 & 8.2 & 0.94 \\
LG 4 & 16 & 112 & 7.0 & 1.12 \\
LG 5 & 20 & 108 & 5.4 & 1.08 \\
LG 6 & 10 & 101 & 10.1 & 0.99 \\
LG 7 & 8 & 98 & 12.3 & 0.93 \\
LG 8 & 13 & 84 & 6.5 & 0.69 \\
LG 9 & 7 & 80 & 11.4 & 0.64 \\
LG 10 & 10 & 65 & 6.5 & 1.06 \\
Avg. & 15.5 & 104.6 & 6.7 & \\
\hline
\end{tabular}

a The average marker spacing was calculated by dividing the summed length of all the linkage groups by the number of marker intervals, while the number of marker intervals represented the difference between the number of markers and the number of linkage groups.

b The number of crossover events per linkage group per individual was estimated by visualizing the haplotypes of the individuals using the GGT program and counting the number of crossovers observed.

Table 5

The average colony diameter of isolates harbouring specific alleles at the loci positioned near putative significant QTL associated mycelial growth.

\begin{tabular}{|c|c|c|}
\hline Alleles $^{\mathrm{a}}$ & $\begin{array}{l}\text { Mean colony diameter } \\
(\mathrm{mm} / \mathrm{wk})^{\mathrm{b}}\end{array}$ & Standard deviation $(\mathrm{mm} / \mathrm{wk})$ \\
\hline \multicolumn{3}{|l|}{ Allelic interactions } \\
\hline het-A1 & $50.7(a)$ & 20.4 \\
\hline het-A2 & 66.6 (b) & 11.3 \\
\hline het-B1 & $58.3(a)$ & 19.9 \\
\hline het-B2 & $59.4(a)$ & 16.3 \\
\hline mat-A1 & $64.2(a)$ & 16.5 \\
\hline mat-A2 & 52.5 (b) & 19.1 \\
\hline mat-B1 & $56.6(a)$ & 19.6 \\
\hline mat-B2 & $61.4(a)$ & 16.4 \\
\hline tc10849-1 & 65.5 (a) & 12.4 \\
\hline tc10849-2 & 50.6 (b) & 19.7 \\
\hline \multicolumn{3}{|l|}{ Digenic interactions } \\
\hline mat-A1, het-A1 & $57.5(\mathrm{ab})$ & 19.5 \\
\hline mat-A2, het- $A 1$ & 43.8 (b) & 19.0 \\
\hline mat- $A 1$, het- $A 2$ & $69.0(a)$ & 6.0 \\
\hline mat- $A 2$, het-A2 & $60.7(\mathrm{ab})$ & 15.0 \\
\hline mat-A1, tc10849-1 & $69.4(a)$ & 11.4 \\
\hline mat-A1, tc10849-2 & $62.7(\mathrm{ab})$ & 15.8 \\
\hline mat-A2, tc10849-1 & $61.5(\mathrm{ab})$ & 15.8 \\
\hline mat-A2, tc10849-2 & 45.1 (b) & 19.7 \\
\hline het-A1, tc10849-1 & $57.9(\mathrm{ab})$ & 18.3 \\
\hline het-A1, tc10849-2 & 46.3 (b) & 9.2 \\
\hline het-A2, tc10849-1 & $71.1(a)$ & 4.2 \\
\hline het-A2, tc10849-2 & $61.1(\mathrm{ab})$ & 9.2 \\
\hline
\end{tabular}

To evaluate allelic and digenic interactions, the growth of isolates with specific alleles or allelic combinations were compared.

b For each comparison the average colony diameters that differ significantly $(P<0.05)$ are indicated by different letters in parentheses.

However, we did detect the presence of digenic interactions that may affect mycelial growth, by analyzing the effects of all possible pair wise allelic combinations of the three markers associated with the significant QTLs on mycelial growth (Table 5). There was a significant difference $(P<0.05)$ in the average mycelial growth of homokaryons that shared the fast growing alleles (mat-A1, het-A2 and tc10849-1) in comparison to homokaryons that shared the alleles associated with slow mycelial growth (mat-A2, het-A1 and tc10849-2). Homokaryons that shared the mat-A1 and het-A2 alleles, the mat-A1 and tc10849-1 alleles or the het-A2 and tc108491 alleles grew faster, while homokaryons that shared the mat-A2 and het-A1 alleles, the mat-A2 and tc10849-2 alleles or the het-A1 


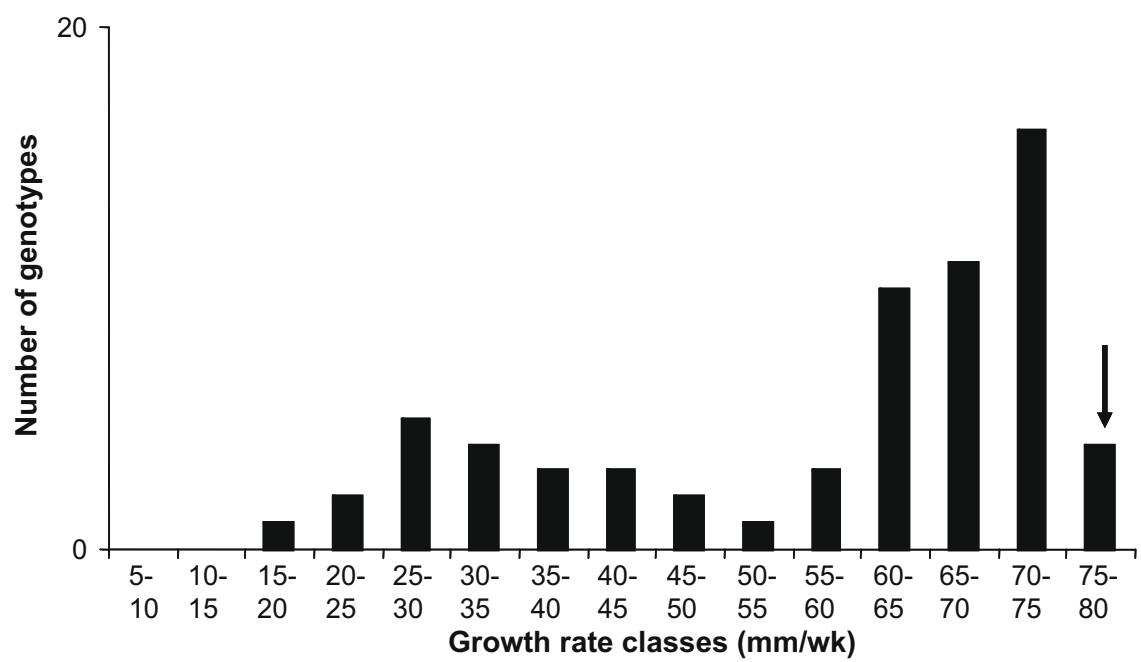

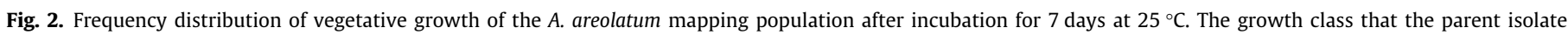
belongs to is indicated with an arrow.

Table 6

QTLs for mycelial growth detected in Amylostereum areolatum using simple interval mapping (SIM) and composite interval mapping (CIM).

\begin{tabular}{llrrl}
\hline Linkage group & Best flanking markers & LOD $^{\mathrm{a}}$ & $R^{2}(\%)^{\mathrm{b}}$ & Significant level $^{\mathrm{c}}$ \\
\hline SIM & & & & \\
Linkage group 2 & ac60482 and het- $A$ & 2.9 & 17 & Significant \\
Linkage group 2 & tc20524 and tc20499 & 2.0 & 12 & Suggestive \\
Linkage group 4 & tc10849 & 2.2 & 13 & Suggestive \\
Linkage group 4 & tc40124 and tc40119 & 1.7 & 10 & Suggestive \\
Linkage group 4 & at60240 & 1.3 & 8 & Suggestive \\
Linkage group 5 & cc20588, mat- A and cc13001 & 1.5 & 9 & Suggestive \\
Linkage group 5 & cc30333 & 1.6 & 10 & Suggestive \\
CIM & & & & \\
Linkage group 2 & ac60482 and het-A & 3.1 & 16 & Significant \\
Linkage group 4 & tc10849 & 2.7 & 11 & Significant \\
Linkage group 4 & tc40124 and tc40119 & 1.5 & 6 & Suggestive \\
Linkage group 4 & at60240 & 1.3 & 6 & Suggestive \\
Linkage group 5 & cc20588, mat- $A$ and cc13001 & 2.8 & 14 & Significant \\
Linkage group 5 & cc30333 & 2.2 & 14 & Suggestive \\
\hline
\end{tabular}

a LOD values were obtained by converting LRS scores using the following equation LRS $=4.6 \times$ LOD $($ Liu, 1998).

$\mathrm{b}$ Explained percentage of phenotypic variance.

c Significance levels determined in Map manager QTX based on a 1000 permutation using $1 \mathrm{cM}$ steps.

and tc10849-2 alleles grew more slowly. Thus, homokaryons that shared one of the alleles (mat-A1, het-A2 or tc10849-1) associated with rapid growth and one of the alleles (mat-A2, het-A1 or tc10849-2) associated with slow growth displayed intermediate growth. Accordingly, homokaryons that shared the mat-A1 and het- $A 1$ alleles, the mat-A2 and het-A2 alleles, the mat-A1 and tc10849-2 alleles, the mat-A2 and tc10849-1 alleles, the het- $A 1$ and tc10849-1 alleles or the het-A2 and tc10849-2 alleles had intermediate growth.

\section{Discussion}

\subsection{A framework map for A. areolatum}

Genetic linkage maps for agaricomycetes have been limited to economically important species such as Agaricus bisporus, Pleurotus ostreatus and H. annosum (Callac et al., 1997; Larraya et al., 2000; Lind et al., 2005). This is because genetic studies for agaricomycetes are hindered by some species being unculturable or due to the inability of their spores to germinate in vitro. Others, including $A$. areolatum, rarely fruit in nature and do not readily produce fruiting bodies in the laboratory (van der Nest et al., 2008). In this study we present a genetic linkage map (Fig. 1) for A. areolatum based on segregation analysis of a population of 80 homokaryons isolated from a basidiocarp collected in the field. For this purpose, we used AFLP analysis, because it provides a rapid means to generate large numbers of markers, allowing us to identify on average 10 polymorphic bands per primer combination (Table 1).

The $A$. areolatum map generated in this study included 10 major (Fig. 1) and 15 minor linkage groups, together spanning about $1693 \mathrm{cM}$. As a related agaricomycete, $H$. annosum, has 10 chromosomes (Lind et al., 2005), it is possible that A. areolatum also has 10 chromosomes, although further research is required to confirm this. Overall, however, the A. areolatum map compares well with those published for other fungi, as the average distance between markers $(8.3 \mathrm{cM})$ is similar to that of $H$. annosum $(6.0 \mathrm{cM})$, Fusarium subglutinans $(12.0 \mathrm{cM})$ and $P$. ostreatus $(5.3 \mathrm{cM})$ (Larraya et al., 2000; Lind et al., 2005; De Vos et al., 2007). The physical distance per unit of recombination for $A$. areolatum $(19.9 \mathrm{kbp} / \mathrm{cM})$ is also comparable to those of $H$. annosum ( $11.1 \mathrm{kbp} / \mathrm{cM}$ ) and Cryptococcus neoformans $(9.6 \mathrm{kbp} / \mathrm{cM})$, although it is considerably lower than those of $P$. ostreatus (35.1 kbp/cM) and C. cinerea (27.9 kbp) (Larraya et al., 2000; Forche et al., 2000; Muraguchi et al., 2003; Lind et al., 2005). Nonetheless, our A. areolatum map is not saturated, as only one of the AFLP markers was positioned within $5 \mathrm{cM}$ of a recognition locus. Future work will seek to place additional markers on this framework map to saturate it further.

Consistent with other agaricomycetes with tetrapolar mating systems (Larraya et al., 2000; Muraguchi et al., 2003), the A. areolatum mat- $A$ and mat- $B$ loci were positioned on separate linkage groups (Fig. 1). The two $A$. areolatum het loci, het- $A$ and het- $B$, also appeared to be positioned on separate linkage groups, which is consistent with previous work (e.g., Kubisiak and Milgroom, 2006). The identification of markers linked to these loci and subsequent characterization of the genes encoded at the recognition loci may be facilitated by approaches such as BSA and map-based cloning. Accordingly, we have found that BSA represents a feasible approach to identify genetic markers closely linked to the recognition loci of $A$. areolatum. It has also been suggested that a relationship between genetic and physical distance $<70 \mathrm{kbp} / \mathrm{cM}$ (compared to our much lower $19.9 \mathrm{kbp} / \mathrm{cM}$ ) is favourable for map-based cloning (Sicard et al., 2003). Therefore, despite the fact that the $A$. 
areolatum map is not yet saturated and possibly does not cover entire chromosomes, it provides the basis for detailed characterization of all the recognition loci of $A$. areolatum using positional cloning.

\subsection{Association between recognition and fitness in A. areolatum}

Mycelial growth in A. areolatum displayed a continuous distribution and, therefore, represents a quantitative trait, consistent with results obtained for other fungi (e.g., Lind et al., 2005; Olson, 2006). Even though mycelial growth is an apparently polygenic trait in A. areolatum, this property seems to be predominantly controlled by only one or a few genes, as is evident from the binomial frequency distribution of mycelial growth rate (Fig. 2) (Buerstmayr et al., 2002). Only three significant putative QTLs and three suggestive putative QTLs for mycelial growth rate were identified in this study, which confirms the presence of only a few genes with major effects and additional genes with minor effects on mycelial growth. Since the six putative QTLs explained $76 \%$ of the total mycelial growth rate variation, it is likely that there are additional QTLS associated with mycelial growth in $A$. areolatum that were not detected. Non-detection of additional QTLs could be due to QTLS being inordinately small to be detected, as was found by Olson (2006). There might also be additional loci not indicated because they were not expressed under the experimental conditions used, or the parents might not have been polymorphic for them.

Three of the four recognition loci of A. areolatum were located in or near the observed QTLs associated with mycelial growth rate (Fig. 1). The het- $A$ and the mat-A loci were positioned in the same region as two of the putative significant QTLs associated with mycelial growth rate in culture, while the mat- $B$ locus was positioned in the same region as one of the putative QTL associated with mycelial growth rate in culture. The association between recognition and mycelial growth rate was confirmed when the growth rate of progeny with different alleles at either their mat- $A$ or het- $A$ loci, differed significantly (Table 5 ). A similar association between mycelial growth and sexual recognition has been observed in $A$. bisporus, P. ostreatus, H. annosum and $S$. commune (Simchen, 1966; Callac et al., 1997; Larraya et al., 2000; Olson, 2006). However, to the best of our knowledge, this is the first time that a link between mycelial growth and self-recognition has been observed in fungi.

\subsection{Genetic determinants of the evolution of the recognition loci}

A large proportion of AFLP markers (29.2\%) displayed significant deviation from the expected Mendelian ratio of parental alleles segregating among the progeny (Fig. 1; Table 2). The concentration of AFLP markers displaying segregation distortion in particular regions suggests that these distortions are the result of biological (both pre- and post-fertilization) mechanisms and not due to chance events or scoring errors. For example, differences among progeny in spore survival, germination and vegetative growth may result in biased selection of more fit individuals for map construction, which in turn may result in markers displaying segregation distortion (e.g., Larraya et al., 2000; Lind et al., 2005).

All of the recognition loci (het- $A$, mat- $A$, mat- $B$ ) associated with growth rate QTLs in $A$. areolatum were positioned near markers or marker clusters showing segregation distortion (Fig. 1). It has been suggested that an association between mycelial growth rate and the mat loci in P. ostreatus, $H$. annosum and $C$. neoformans could potentially explain the segregation distortion displayed at the mat loci or the markers surrounding them (Larraya et al., 2001; Marra et al., 2004; Lind et al., 2005). In the P. ostreatus model system, it has been observed that faster-growing monokaryons belong to specific mating-types (Larraya et al., 2001). A possible explana- tion for the association between mycelial growth and the recognition loci may lie in the fact that the evolutionary forces acting on these systems differ from those acting on the rest of the genome as balancing selection and suppressed recombination play a significant role in their evolution (e.g., Meyer and Thomsen, 2001; Takebayashi et al., 2004; Uyenoyama, 2005).

Balancing selection that retains alleles at approximately equal proportions in populations may help to explain the association between the recognition loci and mycelial growth in A. areolatum. Larraya et al. (2001) also hypothesized that balancing selection acting on the recognition loci of $P$. ostreatus provides a possible explanation for the segregation distortion observed at the mat loci of this fungus. This is because balancing selection, which is thought to maintain the highly polymorphic multi-allelic nature of the recognition loci and the even distribution and long persistence of alleles in populations (Meyer and Thomsen, 2001), also results in the maintenance of potentially deleterious mutations in a population that would otherwise have been lost due to negative selection (Leppälä et al., 2008). Thus, balancing selection will prevent the loss of alleles associated with slow growth resulting in segregation distortion of these alleles. The presence of alleles for slow growth at the het loci, which are known to encode genes involved in housekeeping and other functions (Saupe, 2000), would therefore influence the fitness of individuals negatively. In the case of the mat loci, balancing selection could also affect genes that play no apparent role in mating but that have become entrapped at the mat loci (e.g., Uyenoyama, 2005; Leppälä et al., 2008). This is especially true as some of them encode proteins vital for growth such as ketopantoate reductase involved in energy metabolism and that is encoded at the agaricomycetes mat-A locus (James et al., 2004; Niculita-Hirzel et al., 2008). Therefore, it is likely that mycelial growth and fitness in $A$. areolatum is influenced by balancing selection acting on its recognition loci, or the genomic regions flanking these loci.

Suppressed recombination is a general feature of the recognition loci in eukaryotes where it is thought to limit potentially harmful recombination events such as vegetative self incompatibility or sexual self compatibility (e.g., Awadalla and Charlesworth, 1999; Callac et al., 1997; Meyer and Thomsen, 2001; Uyenoyama, 2005; Menkis et al., 2008). For example, recombination is suppressed between the 25 -kbp region spanning the $u n-24$ and het- 6 loci in N. crassa, as recombination at these loci may lead to vegetative self incompatibility (Smith et al., 2000). In fungi with tetrapolar mating systems where the mat loci consist of multiple sub-loci, recombination within sub-loci is suppressed to prevent self-compatibility, while recombination between different sub-loci is allowed for the generation of new mating type specificities (Lukens et al., 1996). Recombination is apparently also not suppressed in the genomic regions surrounding the agaricomycete mat-A locus, as there is a marked difference in nucleotide diversity between the mat loci and the surrounding regions (James et al., 2006). Recombination thus separates the mat loci that are under balancing selection from the surrounding regions that are under neutral selection (James et al., 2006). Our results suggest that recombination is also not suppressed in the genomic region surrounding the recognition loci of $A$. areolatum, as none of these loci are associated with an excess of markers (e.g., Kubisiak and Milgroom, 2006).

Even though recombination does not appear to be more suppressed at the recognition loci of $A$. areolatum, the possibility that recombination in this fungus is suppressed over the entire genome cannot be excluded. For example, the average observed number of crossovers per linkage group (1.06) for A. areolatum is low, although it is dependent on linkage group size which, in our study, probably did not cover full chromosomes. Nevertheless, the average observed number of crossovers per linkage group in $A$. areolatum is similar to that of $P$. ostreatus (0.89), a fungus known to be 
affected by genome-wide suppressed recombination (Larraya et al., 2000). These values are much lower than the expected theoretical number of crossovers per linkage group for other fungi (e.g., 1.8 for $P$. ostreatus, 1.9 for Fusarium verticillioides and 1.4 for Fusarium graminearum) that are not affected by genome-wide suppressed recombination (Larraya et al., 2000; Jurgenson et al., 2002). Notably, several of the A. areolatum linkage groups displayed less than one observed crossover (Table 4), which is less than the number required for proper chromosome disjunction during meioses (Larraya et al., 2000). It is speculated that genome-wide suppressed recombination may be an evolutionary adaptation for the maintenance of heterozygosity in fungi with homothallic and inbreeding life-cycles (e.g., Callac et al., 1997). It is, therefore, conceivable that genome-wide suppressed recombination has a similar function in A. areolatum with its low overall genetic diversity. However, it is important to emphasise that additional research is required to determine the mode of reproduction of this fungus in nature (e.g., sexual vs. asexual and outbreeding $v$ s. inbreeding), as well as to ascertain the level at which suppressed recombination occurs in the $A$. areolatum genome.

\section{Conclusions}

In this study, we were able to construct a genetic linkage map for $A$. areolatum and to identify potential chromosomal regions that harbour the genes involved in sexual and self-recognition. With the aid of BSA, we were able to also identify markers closely linked to these genes as potential candidates for positional cloning. Although agaricomycete mat loci have previously been mapped and characterized (e.g., Larraya et al., 2000), to the best of our knowledge, this is the first time that any basidiomycete het loci have been mapped to specific regions in a genetic linkage map. The results presented therefore, provide an excellent genetic basis for increasing our knowledge of the recognition systems in this unique model agaricomycete with its obligate mutualistic insect association. This study also represents an important step towards identifying the genetic factors influencing fitness in A. areolatum. This emerges from our linkage map that enabled us to identify regions of the genome (QTLs) that contribute to the expression of growth rate as an indicator of homokaryon fitness. These factors probably have important implications for the epidemiology, evolution and population biology of the fungus, which in turn will influence the spread and control of the fungus/insect association. Ultimately the findings presented here will lead to an improved understanding of the complex interactions that apparently exists between the systems governing recognition and fitness in this and other fungi.

\section{Acknowledgments}

We thank the National Research Foundation (NRF), members of the Tree Protection Co-operative Programme (TPCP) and the THRIP initiative of the Department of Trade and Industry (DTI), South Africa for financial support.

\section{References}

Awadalla, P., Charlesworth, D., 1999. Recombination and selection at Brassica selfincompatibility loci. Genetics 152, 413-425.

Bagley, M.J., Anderson, S.L., May, B., 2001. Choice of methodology for assessing genetic impacts of environmental stressors: polymorphism and reproducibility of RAPD and AFLP fingerprints. Ecotoxicology 10, 239-244.

Buerstmayr, H., Lemmens, M., Hartl, L., Doldi, L., Steiner, B., Stierschneider, M. Ruckenbauer, P., 2002. Molecular mapping of QTLs for Fusarium head blight resistance in spring wheat. I. Resistance to fungal spread (Type II resistance). Theor. Appl. Genet. 104, 84-91.
Callac, P., Desmerger, C., Kerrigan, R.W., Imbernon, M., 1997. Conservation of genetic linkage with map expansion in distantly related crosses of Agaricus bisporus. FEMS Microbiol. Lett. 146, 235-240.

Casselton, L.A., 2002. Mate recognition in fungi. Heredity 88, 142-147.

De Vos, L., Myburg, A.A., Wingfield, M.J., Desjardins, A.E., Gordon, T.R., Wingfield, B.D., 2007. Complete genetic linkage maps from an interspecific cross between Fusarium circinatum and Fusarium subglutinans. Fungal Genet. Biol. 44, 701-714.

Falconer, D., 1989. Introduction to Quantitative Genetics, third ed. Longman Group Limited, London.

Forche, A., Xu, J., Vilgalys, R., Mitchell, T.G., 2000. Development and characterization of the genetic linkage map of Cryptococcus neoformans var neoformans using amplified fragment length polymorphisms and other markers. Fungal Genet. Biol. 31, 189-203.

Glass, N.L., Jacobson, D.J., Shiu, P.K.T., 2000. The genetics of hyphal fusion and vegetative incompatibility in filamentous ascomycete fungi. Ann. Rev. Genet. $34,165-186$.

Hackett, C.A., Broadfoot, L.B., 2003. Effects of genotyping errors, missing values and segregation distortion in molecular marker data on the construction of linkage maps. Heredity 90, 33-38.

Hill, J.A., Otto, S.P., 2007. The role of pleiotropy in the maintenance of sex in yeast. Genetics 75, 1419-1427.

Hulbert, S.H., Ilott, T.W., Legg, E.J., Lincoln, S.E., Lander, E.S., Michelmore, R.W., 1988. Genetic analysis of the fungus, Bremia lactucae, using restriction fragment length polymorphisms. Genetics 120, 947-958.

James, T.Y., Liou, S.-R., Vilgalys, R., 2004. The genetic structure and diversity of the A and B mating type genes from the tropical oyster mushroom, Pleurotus djamor. Fungal Genet. Biol. 41, 813-825.

James, T.Y., Srivilai, P., Kuës, U., Vilgalys, R., 2006. Evolution of the bipolar mating system of the mushroom Coprinellus disseminatus from its tetrapolar ancestors involves loss of mating-type-specific pheromone receptor function. Genetics 172, 1877-1891.

Jurgenson, J.E., Zeller, K.A., Leslie, J.F., 2002. Expanding genetic map of Gibberella moniliformis (Fusarium verticillioides). Appl. Environ. Microbiol. 68, 1972-1979.

Kosambi, D.D., 1944. The estimation of map distances from recombination values. Ann. Eugenics 12, 172-175.

Kubisiak, T.L., Milgroom, M.G., 2006. Markers linked to vegetative incompatibility(vic)genes and a region of height heterogeneity and reduced recombination near the mating type locus (MAT) in Cryphonectria parasitica. Fungal Genet. Biol. 43, 453-463.

Lander, E.S., Green, P., Abrahamson, J., Barlow, A., Daly, M.J., Lincoln, S.E., Newburg, L., 1987. MAPMAKER: an interactive computer package for constructing primary genetic linkage maps of experimental and natural populations. Genomics 1, 174-181.

Larraya, L.M., Idareta, E., Arana, D., Ritter, E., Pisabarro, A., Ramirez, L., 2002. Quantitative trait loci controlling vegetative growth rate in the edible basidiomycete Pleurotus ostreatus. Appl. Environ. Microbiol. 68, 11091114.

Larraya, L.M., Pérez, G., Ritter, E., Pisabarro, A.G., Ramirez, L., 2000. A genetic linkage map of Pleurotus ostreatus. Appl. Environ. Microbiol. 66, 5290-5300.

Larraya, L.M., Pérez, G., Iribarren, I., Blanco, J.A., Alfonso, M., Pisabarro, A.G., Ramirez, L., 2001. Relationship between monokaryotic growth rate and mating type in the edible basidiomycete Pleurotus ostreatus. Appl. Environ. Microbiol. 67, 3385-3390.

Leppälä, J., Bechsgaard, J.S., Schierup, M.H., Savolainen, O., 2008. Transmission ratio distortion in Arabidopsis lyrata: effects of population divergence and the S-locus. Heredity 100, 71-78.

Lind, M., Stenlid, J., Olson, A., 2007. Genetics and QTL mapping of somatic incompatibility and intraspecific interactions in the basidiomycete Heterobasidion annosum s.l.. Fungal Genet. Biol. 44, 1242-1251.

Lind, M., Olson, A., Stenlid, J., 2005. An AFLP-marker based genetic linkage map of Heterobasidion annosum locating intersterility genes. Fungal Genet. Biol. 42, 519-527.

Liu, B., 1998. Statistical Genomics: Linkage, Mapping and QTL Analysis. CRC Press, Boca Raton, FL.

Lukens, L., Yicunt, H., May, G., 1996. Correlation of genetic and physical maps at the A mating-type locus of Coprinus cinereus. Genetics 144, 1471-1477.

Marra, R.E., Huang, J.C., Nielsen, E., Fung, K., Heitman, J., Vilgalys, R., Mitchell, T.G., 2004. A genetic linkage map of Cryptococcus neoformans variety neoformans serotype D (Filobasidiella neoformans). Genetics 167, 619-631.

Manly, K.F., Cudmore, R.H., Meer, J.M., 2001. Map manager QTX, cross-platform software for genetic mapping. Mamm. Genome. 12, 930-932.

Menkis, A., Jacobson, D.J., Gustafsson, T., Johannesson, H., 2008. The mating-type chromosome in the filamentous ascomycete Neurospora tetrasperma represents a model for early evolution of sex chromosomes. Plos. Genet. 4, e1000030.

Meyer, D., Thomsen, G., 2001. How selection shapes variation of the human major histocompatibility complex: a review. Ann. Hum. Genet. 65, 1-26.

Muraguchi, H., Ito, Y., Kamada, T., Yanagi, S.O., 2003. A linkage map of the basidiomycete Coprinus cinereus based on random amplified polymorphic DNAs and restriction fragment length polymorphisms. Fungal Genet. Biol. 40, 93-102.

Myburg, A.A., Remington, D.L., O’Malley, D.M., Sederoff, R.R., Whetten, R.W., 2001. High-throughput AFLP analysis using infrared dye-labelled primers and an automated DNA sequencer. BioTechniques 30, 348-357.

Nauta, M.J., Hoekstra, R.F., 1994. Evolution of vegetative incompatibility in filamentous ascomycetes. I. Deterministic models. Evolution 48, 979-995. 
ARTICLE IN PRESS

10

M.A. van der Nest et al./Fungal Genetics and Biology $x x x$ (2009) $x x x-x x x$

Niculita-Hirzel, H., Labbé, J., Kohler, A., La Tacon, F., Martin, F., Sanders, I.R., Kües, U., 2008. Gene organization of the mating type regions in the ectomycorrhizal fungus Laccaria bicolor reveals distinct evolution between the two mating type loci. New Phytol. 180, 329-342.

Olson, A., 2006. Genetic linkage between growth rate and the intersterility genes $\mathrm{S}$ and $\mathrm{P}$ in the basidiomycete Heterobasidion annosum s. lat. Mycol. Res. 110, 979-984.

Remington, D.L., Whetten, R.W., Liu, B.H., O’Malley, D.M., 1999. Construction of an AFLP genetic map with nearly complete genome coverage in Pinus taeda. Theor. Appl. Genet. 98, 1279-1292.

Saupe, S.J., 2000. Molecular genetics of heterokaryon incompatibility in filamentous ascomycetes. Microb. Mol. Biol. Rev. 64, 489-502.

Shibata, T., 2001. Functions of homologous DNA recombination. RIKEN Rev. 41 21-23.

Sicard, D., Legg, E., Brown, S., Babu, N.K., Ochoa, O., Sudarshana, P., Michelmore, R.W., 2003. A genetic map of the lettuce downy mildew pathogen, Bremia lactucae, constructed from molecular markers and avirulence genes. Fungal Genet. Biol. 39, 16-30.

Simchen, G., 1966. Monokaryotic variation and haploid selection in Schizophyllum commune. Heredity 21, 241-263.

Slippers, B., Coutinho, T.A., Wingfield, B.D., Wingfield, M.J., 2003. A review of the genus Amylostereum and its association with woodwasps. SAJS 99, 70-74.

Smith, M.L., Micalli, Q.C., Hubbard, S.P., Mir-Rashed, N., Jacobson, D.J., Glass, N.L., 2000. Vegetative incompatibility in the het- 6 region of Neurospora crassa is mediated by two linked genes. Genetics 155, 1095-1104.
Staelens, J., Rombaut, D., Vercauteren, I., Argue, B., Benzie, J., Vuylsteke, M., 2008. High-density linkage maps and sex-linked markers for the black tiger shrimp (penaeus monodon). Genetics 179, 917-925.

Takebayashi, N., Newbigin, E., Uyenoyama, M.K., 2004. Maximum-likelihood estimation of rates of recombination within mating-type regions. Genetics 167, 2097-2109.

Thomas, W.T.B., Baird, E., Fuller, J.D., Lawrence, P., Young, G.R., Ramsay, L., Waugh, R., Powell, A.J., 1998. Identification of a QTL decreasing yield in barley linked to Mlo powdery mildew resistance. Mol. Breed. 4, 381-393.

Uyenoyama, M.K., 2005. Evolution under tight linkage to mating type. New Phytol. $165,63-70$.

Van Berloo, R., 1999. GGT: software for the display of graphical genotypes. J. Hered. 90, 328-329.

Van der Nest, M.A., Slippers, B., Stenlid, J., Wilken, P.M., Vasaitis, R., Wingfield, M.J., Wingfield, B.D., 2008. Characterization of the systems governing sexual and self-recognition in the white rot Agaricomycete Amylostereum areolatum. Curr. Genet. 53, 323-336.

Van Ooijen, J.W., Voorrips, R.E., 2001. JoinMap 3.0 Software for the Calculation of Genetic Linkage Maps. Plant Research International, Wageningen, The Netherlands.

Vos, P., Hogers, R., Bleeker, M., Reijans, M., Van der Lee, T., Hornes, M., Frijters, A., Pot, J., Peleman, J., Kuiper, M., Zabeau, M., 1995. AFLP: a new technique for DNA fingerprinting. Nucl. Acids. Res. 23, 4407-4414.

Worral, J.J., 1997. Somatic incompatibility in basidiomycetes. Mycology 89, 24-36.

Please cite this article in press as: van der Nest, M.A., et al. Genetic linkage map for Amylostereum areolatum reveals an association between vegetative

growth and sexual and self-recognition. Fungal Genet. Biol. (2009), doi:10.1016/j.fgb.2009.06.002 